\title{
Zur Behandlung der Extremitäten-Schußfrakturen mit der Schraubenextensionsschiene.
}

Von Sanitätsrat Dr. Heinz Wohlgemuth, z. Z. Leitender Chirurg am Reservelazarett Berlin-Steglitz.

In Nr. 25 (1915) der D. m. W. habe ich eine Schraubenextelisionsschiene für die Schußfrakturen der Extremitäten angegeben, die sich, wie ich von vielen Seiten aus Kriegs̀- und Heimatlazaretten hörte, gut, in gewissen Fällen besser als die bisher bekannten Apparate und geübten Methoden bewährt hat. Mein Hauptaugenmerk war darauf gerichtet, daß die Schiene zunächst die beiden Grundforderungen für ein gutes Resultat erfüllte, neben guter Lagerung der Extremität absolute Ruhigstellung der beiden benachbarten Gelenke und gute, in jedem Augenblick zu modifizierende Extension. Dabei sollte sie die Möglichkeit geben, den Patienten vom Bett auf den Verbandsłisch ohne Störung der Extension, ohne Verschiebung der Bruchenden und ohne Schmerzen zu transportieren, freies Feld für den Verbandwechsel und für notwendige Eingriffe an der Wunde zu lassen und nicht zuletzt einen Druckbrand bei noch so starker Extension zu vermeiden. Alle Anforderungen erfüllte die bisher von mir angewandte und 1 . c. beschriebene Methode, bis auf die letzte. Leider mußte ich in einigen Fällen bei durch starken Blutverlust und lange Eiterung heruntergekommenen Kranken bei der Oberschenkelschiene einen Druckbrand am Schambein oder an Kalkaneus in Kauf nehmen. Auch bei den ganz hohen Oberschenkelschußbrüchen war die Schiene nach der derzeitigen Publikation nicht zu verwenden. Meine dahingehenden Versuche waren noch nicht abgeschlossen, und mit Recht macht v. Stubenrauch in seiner Arbeit über die Behandlung der Oberschenkelschußfrakturen in Nr. 15 der M. m. W. 1916 auf diesen Mangel aufmerksam.

Seit einigen Monaten habe ich nun die Schiene modifiziert und feststellen können, daß sie auch diese letzten Forderungen erfüllt, sodaß ich glaube, sie nun in ihrer jetzigen Form uneingeschränkt bei jeder Art von Schußbruch der unteren Extremität empfehlen zu können. Sie wird meines Erachtens gerade für die ganz hohen Oberschenkelschuß. brüche von besonderem Wert sein.

Für den, der sie sich vorkommenden Falles in kurzer Zeit selber zurechtbiegen will, gebe ich folgende Anweisungen:

Von dem 1 Zoll breiten, 3-4 mm starken verzinkten Bandeisen wird ein $290 \mathrm{~cm}$ langes Stück abgeschnitten. An dem Schenkel, der der Innenseite des Beines anliegen soll, wird, vom Endpunkt abwärts gerechnet $55 \mathrm{~cm}$, die Schiene rechtwinklig über die Kante. umgebogen, der Winkel wird mit einigen Hammerschlägen flach geschlagen. Dieser $55 \mathrm{~cm}$ lange Schenkel wird wieder so rechtwinklig in Höhe von $15 \mathrm{~cm}$ über die Kante abgebogen, daß das letzte $40 \mathrm{~cm}$ lange Ende flach über dem Bauch liegen kann.

Nun wird vom Fuß des vertikalen $15 \mathrm{~cm}$ langen Streifens nach abwärts in einer Entfernung von $90 \mathrm{~cm}$ das Band rechtwinklig über die Fläche, wieder in einem Abstand von $15 \mathrm{~cm}$ nochmals rechtwinklig

1) M. m. W. Nr. 1 S. $16 .{ }^{2}$ ) M. m. W. Nr. 8. 
Fig. 1.

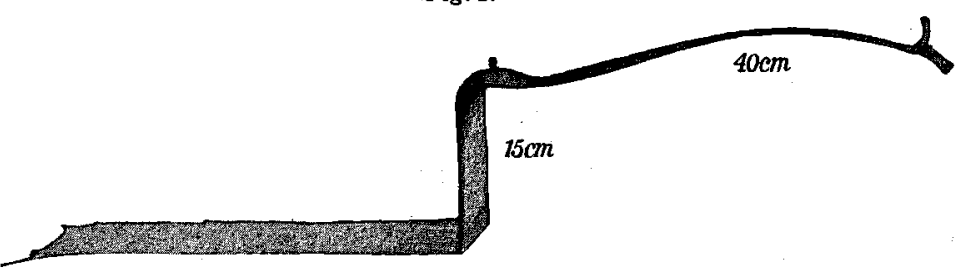

über die Fläche abgeknickt, und das Modell der. Schiene ist fertig. Die beiden freien Enden der Schiene, die am Thorax eingegipst werden sollen, werden noch auf 3-4 cm Länge mit einigen Meißelschlägen gespalten, eines dieser gespaltenen Enden rechtwinklig aufgebogen, um einem Hochrutschen der Schiene im Gipsverband Gegengewicht zu geben und sie besser zu fixieren. Das $15 \mathrm{~cm}$ lange Fußstück wird gelocht für die Extensionsschraube.

Fig. 2.

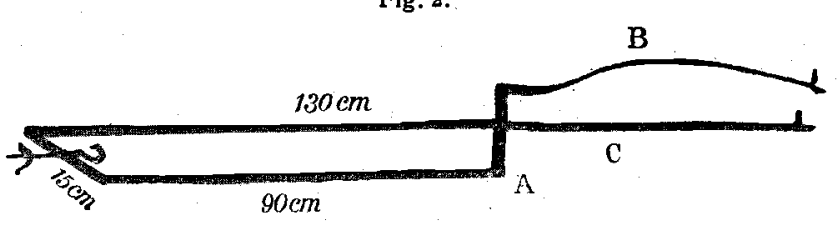

Nun wird die Schiene dem auf flachem Tisch gelagerten Patienten angepaßt. Sie muß folgendermaßen liegen: Der Winkel A ist mindestens $10 \mathrm{~cm}$ vom Schambein entfernt. Der Schenkel B wird zweckmäßig wie in Fig. 2 so modelliert, daß er gewölbt über dem Unterbauch liegt und nur mit seinem letzten Ende dem Hypochondrium flach und ohne Druck aufliegt, und soweit nach der Körpermitte hin abgebogen, daB er über die Mittellinie hinausragt. Diese Stellung garantiert eine leichte $\mathrm{Ab}$ duktion des Beines. Der $130 \mathrm{~cm}$ lange Schenkel C wird (über einer Stuhlkante) so modelliert, daß er den Konturen des oberen Abschnitts des Oberschenkels und des Beckens entspricht, freien Raum für den Verband und die Wundbehandlung an den entsprechenden Stellen läßt und daß sein freies oberes Ende glatt und ohne Druck der Flanke und dem Thorax anliegt. Jetzt kann der Verband gemacht werden, in fol: gender Reihenfolge:

1. Anlegung eines Mastisol-Extensionsstreifens (Polsterung der Malleolengegend) so weit wie möglich über das Knie hinaus. Hierzu eignet sich am besten ein $8-10 \mathrm{~cm}$ breiter Barchentstreifen, der an seinen freien Enden gespalten werden kann, um so den Oberschenkel besser zu umgreifen, ein Verfahren, das sich besonders bei kleineren Nebenverwundungen der Weichteile empfiehlt, die man dadurch für die Wund. versorgung frei halten kann.

2. Umwicklung des noch einmal mit Mastisol bepinselten Ex tensionsstreifens mit gutsitzender Mull- oder Flanellbinde, von den Zehen beginnend, nicht zu fest und bei rechtwinkliger Stellung des Fußes.

3. Wundverband.

4. Gute Lagerung des Patienten auf Beckenstützen, möglichst horizontal Unterstützung des Kopfes und der Schultern in gleicher Höhe wie Becken und Beine, die in leichter Abduktion gehalten werden. Ein wicklung des Rumpfes von den Brustwarzen bis etwa über die Hälfte des Beckens herab in breite Mullbinden. Darüber gute dicke Polsterung mit Watte mit besonderem Schutz der Spina anterior superior und der unteren Rippenbögen. Nochmalige Mullbindeneinwicklung.

5. Erste Gipsbindenumwicklung des Thorax. Von den Brustwarzen bis zum Becken. Gleichmäßig etwa sechs bis acht Iagen übereinander.

6. Nach nicht zu geringer Erhärtung dieses Gipsbettes Anlegen der Schiene ohne Druck (Abstand des inneren Schenkels vom Schambein!). Die beiden freien Enden müssen sich nun glatt einerseits der Flanke, anderseits dem Bauch und dem unteren Thoraxabschnitt an legen. Sie werden von einem Assistenten ganz leicht angedrückt; das geschlossene Ende wird mit dem Fuß zugleich in Höhe der Ferse gehalten.

7. Zweite Gipsbindeneinwicklung mit guter Fixation der freien Schienenenden. Die senkrecht aus dem Gipsverband hervorstehenden umgebogenen Enden werden freigelassen. Sechsfache gleichmäßige Lage wird hier genügen.

8. Das Bein wird mit einem unter dem Knie und oberhalb der Malleolen durchgeführten Bindenzügel angehoben, um die Lagerungshängematte von einem Schienenschenkel zum anderen mit einer kräftigen, etwa $10 \mathrm{~cm}$ breiten Binde so anzulegen, daß das Knie nicht nach unten durchgedrückt werden kann.

9. Der Extensionsschraubhaken wird am Fußbrettchen des Extensionsstreifens, das mit einem Haken oder einer starken Bindfadenöse versehen ist, eingehakt und nur soweit angezogen, daß die Extremität ruhig liegt. Die notwendige Extension findet erst am folgenden Tage

nach vollkommener Erhärtung des Gipsverbandes und Festwerden des Mastisolzugstreifens statt.

10. Umwicklung des ganzen Beines und der Schiene mit Bindentouren wie bei einer Hohlschiene zur Ruhigstellung.

Am folgenden Tage Entfernung dieser Umwicklung und Extension. Eine etwaige Abweichung der Bruchenden in der Längsachse wird durch Zug und Gegenzug mit einigen Bindentouren um den einen und um den anderen Schienenschenkel, eine winklige Abknickung mit fester Anpressung an einen Schienenschenkel ausgeglichen.

Ich halte es nicht für erforderlich, habe jedoch in einigen Fällen davon Gebrauch gemacht, einen Gegenzug in der Schenkelbeuge auf einer oder beiden Seiten anzulegen. Zu diesem Zweck habe ich Trikotschlauchbinden, in Ermangelung derselben breite, zu einem Schlauch zusammengenähte Flanellbinden von $1 \mathrm{~m}$ Länge, in der Mitte $30-40 \mathrm{~cm}$ weit mit Polsterwatte ausgestopft, sie in dem Korsett so eingegipst, da $B$ zunächst ihre Enden über das Gipskorsett nach oben hinausragen, dann umgeschlagen und mit den nächsten Touren befestigt. Sie werden zweckmäBig vorher so gedehnt, daß ihre Elastizität nicht mehr groß ist. Statt dieser Gegenzüge kann man, was sich bei flachem zylindrischen Thorax empfehlen dürfte, mit dem Anlegen des Rumpfgipsverbandes zugleich den anderen Oberschenkel bis fast zum Knie mit eingipsen und in der Schenkelbeuge tief ausschneiden. Hierdurch wird eine vollkom. mene Ruhigstellung mit Sicherheit erzielt.

Fernere Ausschnitte in dem Gipskorsett können eventuellen $\mathrm{De}$ kubitus am Kreuzbein oder Wunden an der Brust und am Rücken zur Versorgung freilassen.

Sollte es nicht möglich sein, eine Mastisolzugbinde anzulegen, so wird der FuB in einen Gipsschuh, wie in Nr. 25 der D. m. W. 1915 beschrieben, gelegt. (Figg. 3 und 4.)

Auch die Oberarmextensionsschiene habe ich etwas modifiziert. Der mediale Schenkel, der bisher, etwas umgebogen, sich gegen ein ge. polstertes Gipsbett in der Achselhöhle stützte, liegt jetzt dem Thorax an. Bei der vorsichtigsten Polsterung konnte ein Druck auf die Gefäße

Fig. 3.
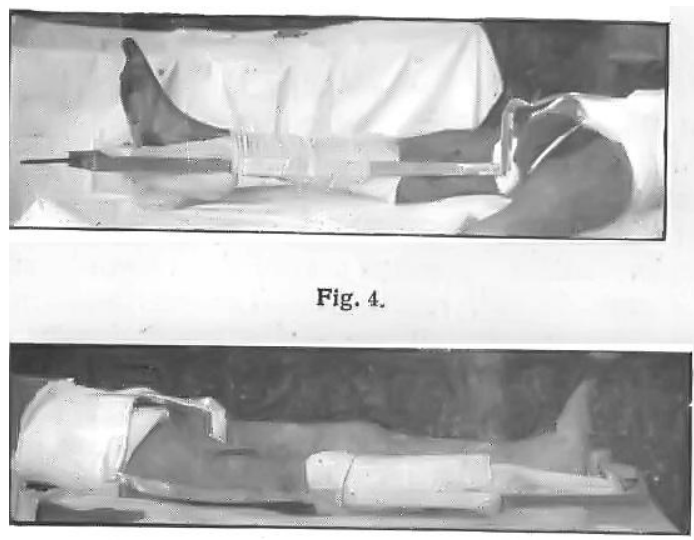

und Nerven nicht immer vermieden werden, und es kam ein Oedem des Armes zustande. Die Schiene von $1 \mathrm{~m}$ Länge ist jetzt so zurechtgebogen, daß sie mit ihrem äußeren Schenkel dem Gipsbett auf der Schulter, mit ihrem medialen, $15 \mathrm{~cm}$ abwärts vom Ende im Winkel von $45^{\circ}$ abgebogenen Schenkel dem Thorax anliegt. Vor dem Anlegen wird sie in der Schuß. höhe zur freien Wundbehandlung ausgebogen.

Die Anlegung geschieht folgendermaßen:

1. Wundversorgung des Oberarmschusses.

2. Anlegen der Ellbogen-Gipsmanschette mit Extensionsschlinge in rechtwinkliger Stellung des Vorderarms und Mittelstellung zwischen Supination und Pronation. Gute Polsterung der Epikondylen und der Ellbogenbeuge.

3. Gute Polsterung, Watteumwicklung der Schulter, der Achselhöhle und des Thorax bis zum Rippenbogen. Bindenumwicklung.

4. Erste Gipsbindeneinwicklung von Schulter und Thorax. Acht Lagen.

5. Nach mäßigem Erstarren Anlegen der Schiene, die wie die Oberschenkelschiene an den Enden eingekerbt und aufgebogen ist.

6. Zweite Gipsbindeneinwicklung mit guter Fixierung der Schiene. 7. Mitella.

Die Schienen werden im Handel zu haben sein. Den Feld- und Kriegslazaretten werden sie rom Haupt-Sanitäts-Depot auf Anfordern zugeschickt. (Fig. 5.) 